\section{IL CASO LELLA COSTA}

THE CASE “LELLA COSTA”
Nel panorama teatrale italiano, Lella Costa si distingue come un caso particolare: non solo per il suo genere, per la sua femminilità che tuttavia non svilisce la sua comicità pungente ed efficace - nonostante la tradizione teatrale sia lenta a cambiare opinione quando si riferisce alla comicità femminile, per cui si preferisce parlare di comicità asessuata o universale -; ma soprattutto per il suo impegno politico-sociale che trapela nei suoi brillanti monologhi comici e l'attenzione che catalizza su tem seri di attualità, gli stessi temi che tratta con una leggerezza e un'apparente frivolezza sulla base della quale sembra aver cucito e costruito i suoi spettacoli. Con la stessa apparente noncuranza e frivolezza riesce a "spostare" l'alone di seriosità che incombe su tematiche sociali di un certo peso, e a farli percepire "straniati" per operare quel distanziamento necessario alla coscienza analitica e critica: in tal modo, dunque, similmente alla lezione del giullare medioevale recuperato dall'attività filologica e teatrale di Dario Fo, l'autrice comica, come nella vecchia tradizione dei giullari popolari dell'epoca medioevale, riesce a lanciare delle proposte e iniziative sociali e a trasmettere e stimolare prese di posizione in ambito politico e culturale.

Un giullare moderno, e per giunta donna, che fa ricorso alle maschere non solo letterarie, con una retorica personalissima ben definita, che fa uso degli abiti e degli accessori anche allo scopo di attirare l'attenzione del pubblico, travestendosi, in altre parole, usando una serie di maschere, che man mano personalizza, fino a diventare il personaggio "Lella Costa". L'altro elemento di distinzione della sua teatralità è la scelta del monologo come tipo di discorso e cornice per la sua comicità e questo aspetto richiede un approfondimento a parte.

\section{Il "caso" del monologo}

La scelta del monologo da parte di un interprete-autore, come fa notare Puppa rimanda alla tradizione dei nostri comici dell'arte ed anche alla serata d'onore del mattatore ottocentesco, e che nel panorama artistico italiano continua ad essere un tipo di spettacolo molto apprezzato agevole per i costi, e per le tournées, cosa che giustifica perché nel teatro si assiste a un sempre maggiore interesse al monologo (Puppa 2002: 445). E non è solo per l'evidente vantaggio economico di rappresentare un'opera teatrale con un solo personaggio, pochi oggetti, una messa in scena semplice, minimalista. Molti attori che sono anche gli autori dei propri testi privilegiano questo genere anche per altre ragioni e non solo per motivi di narcisismo: nell'attualità il monologo è anche la forma preferita dal teatro politico, un teatro che più che utilizzare la memoria individuale rimanda a questioni storico-sociali, anche se sotto forma comica. Altri autori-attori scelgono la voce monologante come cornice della narrazione, 
un protagonista su cui ricade il punto di vista dei fatti che si vuole assolutamente parziale, per "istrionismo attorico" se preso sul serio; ma lo stesso motivo giustifica la sua inversione nel comico con la parodia. Il monologo comico, finalizzato al puro divertimento è una forma paraletteraria molto popolare diffusa nelle trasmissioni televisive, e soprattutto per questo motivo, nei nostri anni si può considerare la formateatrale preferita dagli autori-attori emergenti, ma anche di quelli consacrati. La sua struttura è in stretta relazione con i generi popolari, per i modi in cui le sue tecniche econvenzioni sono passate anche ad altre forme dei mezzi di comunicazioni di massa.

Ciò che è avvenuto è un passaggio dalle convenzioni del monologo ai "mass media" e, successivamente, il ritorno del monologo al teatro. I casi i di autori-attori/attrici negli show televisivi attuali, come Ascanio Celestini, Giobbe Covatta, Gianfranco Iannuzzo, Francesca Reggiani, Luciana Littizzetto, Antonio Albanese, Gigi Proietti e Sabina Guzzanti, per citare alcuni tra i più conosciuti, sono esempi della popolarità del monologo al livello dei mezzi di comunicazione di massa. Si tratta di un fenomeno artistico che ha un successo straordinario non solo in Italia: il monologo è diffusissimo negli altri Paesi e nel teatro e nello spettacolo europeo ha sempre più spazio. Il successo di questa forma teatrale è tale che, come osserva Franco Portone:

\section{La televisione, il cabaret con i loro format hanno purtroppo influenzato non pochi autori, che in uno spettacolo anche a più personaggi prediligono ormai il monologo... Si riflette, probabilmente, l'alienazione dei rapporti interpersonali, la difficoltà di comunicazione, l'estremizzazione dell'individualismo, il linguaggio che è surrogato dai segni, per nascondere una debolezza emotiva di fondo. (Portone, 2008)}

Questa stessa difficoltà e incapacità comunicativa e di ascolto per cui lo scambio interpersonale si risolve in monologhi soggettivi, il teatro la ripropone in forma artistica. Ciò spiega anche la forte attrazione per il monologo durante tutto il XX sec. - anche se nel teatro è un fenomeno degli ultimi decenni fondamentalmente - poiché numerosi drammaturghi vedono in esso un modo privilegiato di entrare nella soggettività, di far parlare la soggettività direttamente. (Martín Clavijo, 2009: 18) È uno degli strument più attuali e immediati per restituire all'attore la dimensione che appartiene al proprio bagaglio originario; e restituisce all'uomo o alla donna, la sua necessità di esprimere e condividere la propria esperienza. Si tratta di recuperare storie personali, spesso peculiari, oppure vissuti di persone che hanno molto in comune con noi. Per questo la messa in scena dell'autobiografia finta o non finta è la forma preferita delle giovani drammaturghe contemporanee, soprattutto in Italia.

\section{Il monologo nel teatro}

È la struttura che meglio si presta alla denuncia ridicolizzata di tematiche sociopolitiche come dimostrano Dario Fo, Carmelo Bene, Franca Rame, Beppe Grillo e
Roberto Benigni; ed è anche il genere preferito di quei comici che si sono specializzat nella ridicolizzazione delle manie, ossessioni, e nelle caricature esilaranti di figure o di personaggi politici, ma anche di personaggi comuni, della strada, per puro divertimento.

Il monologo è un discorso che tratta della verità apparente e di quella vera -spesso mascherata dall'intento comico-, parla e occulta il sentimento dei suoi enunciatori, esplora il loro inconscio attraverso il loro pensiero cosciente che quasi sempre libera ossessioni e contrasti irrisolti dei drammaturghi. Per questo, come sottolinea Milagros Martín Clavijo, è uno strumento particularmente utile per la critica sociale e culturale. Anche per questo è il mezzo preferito da quelle autrici, scrittrici, e drammaturghe che cercano di aprirsi uno spazio particolare per esprimersi, per attirare l'attenzione e farsi ascoltare (Martín Clavijo, 2009: 15-17). Alcuni studiosi tra cui Chiara Alessi (2005:14), distinguono almeno tre tipi di monologo:

i monologhi narrativi;

a) i monologhi-personaggio, che si concentrano più sulla figura del locutore che sugli eventi raccontati;

b) il monologo che ha una forma dialogica: si tratta di quei monologhi che apparentemente sono dialoghi, con interlocutori che non sentiamo e non vediamo, o perché riportati con un discorso indiretto, o perché "supposti" dai "metamedia" come li definisce Paolo Puppa, e cioè il telefono, la radio, la televisione (Puppa, 2003:168). Carmelo Bene ritiene che il monologo è un delirio che incorpora, assorbe 1' "altro" con cui deve stabilire un dialogo (Bene, 2002: 1002). Ecco allora che il monologo è il fenomeno che raccoglie una pluralità di "voci", sia interiori - il dialogo con l' "altro" se stesso-sia le "voci" oggettive -quelle degli "altri" personaggi del racconto coinvolti nel dialogo con il soggetto parlante-

Nei monologhi di Lella Costa i personaggi che l'autrice crea con la sua interpretazione dialogano costantemente tra di loro, come rappresentazioni delle sue sfaccettature e anche delle tante tipologie di donne a cui si rivolge: in un certo senso, con queste maschere -spesso rappresentate dai frequenti cambi d'abito- la Costa "ritrae" identità diverse e così facendo coinvolge il suo pubblico femminile per via di uno spettatore implicito che sia il più ampio possibile.

\section{Il monologo di Lella Costa}

Puppa fa notare che la caratteristica dell'attore/attrice autore che recita il suo spettacolo (da lui definito performer), consiste $>$ (Puppa, 2002: 446). Lella Costa, però, non si finge diversa da se stessa e tuttavia sia come autrice riesce a costruirsi un personaggio, sia come attrice diventa il "personaggio di se stessa". Così facendo valorizza i momenti fatici della comunicazione con il pubblico, stabilendo un dialogo con lo spettatore implicito. 
Ad ogni modo si tratta di un dialogo apparente, trattandosi, in realtà, di un monologo, in cui il narratario non ha un peso e una funzione reale: perché un pubblico ben definito supporrebbe un "personaggio Lella Costa" altrettanto definito, con i rischio di fossilizzazione in una "unica maschera", e pertanto, troppo surreale, eterea. Mentre l'esigenza di questa autrice-attrice è di calarsi nel reale e di suscitare credibilità attraverso l'identificazione con la sua realtà e attualità di donna del XX secolo con una precisa storia e responsabilità sociale.

Nella costruzione del suo personaggio "donna-con-cui-identificarsi", la Costa crea, dunque, un personaggio dalle mille sfaccettature rappresentate dalle tante maschere quanti sono i travestimenti che adotta, proprio per "deviare" l'attenzione rispetto al personaggio "Lella Costa", perché un rapporto dialogico con un pubblico ben definito comprometterebbe la sua possibilità di rivolgersi a più fasce possibili di pubblico e soprattutto a più tipologie di donne, per poter "raccontare" la storia di queste donne e aiutarle a prendere coscienza e distanza dalle loro nevrosi, ironizzarci e riderci sopra, come sembra essere la sua preoccupazione principale. Lo spostamento di sguardo che promuove con questi suoi personaggi, infatti, sono alla base dell'ironia che non serve solo a divertire, ma a fornire una chiave di conoscenza di noi stessi/e e per comprendere meglio il mondo e ciò che succede intorno. Ecco perché, per l'autrice interprete è necessario raccontare, per capire se stessa, i suoi vari "io", per capire gli altri e le altre e per condividere con loro le proprie esperienze. E il monologo-narrazione in teatro permette di farlo meglio di altre forme artistiche.

\section{Lella Costa comica dell'Arte}

Se negli ultimi tempi, come spesso si dice, la tendenza nel teatro è quella di privilegiare il monologo come espressione e cornice che elimina le ricerche sullo spazio (dove inscenare), sul come (i segni dello spettacolo) e sul quando e quanto (orari e costi della rappresentazione) perché ormai tutta l'attenzione si concentra sul cosa dire, come rileva Paolo Puppa (2002: 449); se questa tendenza che ha decretato un nuovo genere di narrazione definito "teatro narrazione" o "teatro racconto", ha suscitato una certa inquietudine e allarme che ha fatto parlare da più parti di "morte del teatro"; ci sembra di poter dire che Lella Costa ha contribuito, con la sua esperienza e la sua formula spettacolare di affabulatrice comica, a recuperare la tradizione tipicamente italiana, sia attraverso l'uso dei travestimenti e delle maschere, sia attraverso la sua comicità peculiare. Le sue maschere sono prodotte dalla caratterizzazione di personaggi che avviene per la gestualità, per le espressioni assunte, per gli accenti e per i registri linguistici specifici. Ed ecco che nascono le varie tipizzazioni (maschere) della soubrette ammaliante e ironica, del produttore cinematografico romano cinico e superficiale, del giornalista invadente, della centralinista ipercortese e impersonale, del bancario svizzero, ecc..

I suoi spettacoli sono il risultato di una tecnica di montaggio di immagini, di racconti, di digressioni, di associazioni (in cui si riconoscono intere generazioni) e di racconti autobiografici-condivisi però da molte donne-, che rispondono ad un assemblaggio drammaturgico e recitativo che ricorda tanto lo stile dei giullari rivisitato in chiave moderna. Questa figura di giullare-affabulatrice e allo stesso tempo interprete-narratrice "impegnata" della nuova generazione, costituisce un modello di riferimento militante e forse anche culturale, nei tempi del disamore politico e del crollo delle ideologie.

Il modello, come confessa la stessa autrice, era lo stile affabulatorio di Dario Fo che, per capire ciò che succedeva nella realtà quotidiana, inventava storie dal palcoscenico, perfino costruendo dei vangeli apocrifi, ma sempre rispettando le regole della finzione. Ma ciò che fa pensare ad un "Caso Lella Costa" è la capacità straordinaria di questa autrice-interprete di superare, con i suoi spettacoli, la funzione di testimoniare sui tempi attuali, attraverso l'intrattenimento, per giungere, con la narrazionedivagazione, alla poesia e al canto, perché i suoi testi, che spesso raccontano di problemi e temi attuali trasfigurati attraverso miti e storie antiche (la guerra, la difficile relazione uomo-donna, l'educazione sentimentale mancata, ecc.) soprattutto gli ultimi -scritti insieme a Gabriele Vacis - costituiscono una specie di partitura, con dei ritmi e delle scansioni veramente musicali.

\section{Storia di Lella Costa}

Lella Costa debutta nel 1987 con il primo spettacolo di cui è anche autrice, Adlib, a cui seguiranno nel 1988 Coincidenze, e nel 1990 Malsottile. I tre monologhi citati saranno raccolti nel 1995, nella raccolta intitolata La daga nel Loden. Del 1992 è il monologo Due; nel 1994 scriverà Magoni (con musiche originali di Ivano Fossati); mentre il 1996 è la volta del monologo Stanca di guerra (testo scritto con la collaborazione di Alessandro Baricco). Il 1998 è l'anno di Un'altra storia, con la regia di Gabriele Vacis: i tre monologhi citati - Due, Magoni e Un'altra storia - saranno pubblicati da Feltrinelli nel volume Che faccia fare. Del 2000 è il monologo Precise parole: gli ultimi quattro monologhi, insieme ai primi tre già pubblicati con il titolo La daga nel Loden, usciranno nell'anno 2002 con l'edizione Feltrinelli nel volume, In tournée.

In seguito, la Costa, nel 2002, scrive Traviata; nel 2005 Alice, una meraviglia di Paese con la regia di Giorgio Gallione; del 2007 è il monologo Amleto: gli ultimi tre testi saranno pubblicati da Feltrinelli nel libro, Amleto, Alice e la Traviata, con la prefazione di Michele Serra. Nel 2009 ha debuttato con Ragazze, nelle lande scoperchiate del fuori, spettacolo con il quale è attualmente in tournée.

Per questo studio mi sono basata soprattutto sulla retorica del suo discorso così come appare nella raccolta dei suoi primi tre monologhi raccolti nell'edizione del libro La Daga nel Loden, con riferimenti ad interviste pubblicate e rintracciabili nel sito internet della sua casa di produzione: IRMA. Si veda: http:// www.irmaspettacoli.it/lella_autrice.html

\section{La retorica comica "femminile"}

Lella Costa dichiara più volte la sua ammirazione per Franca Valeri e per il suo tipo di comicità, riferendosi spesso alla Valeri come modello di attrice brillante -seppur distanziandosene, in parte, ironicamente quando le attribuisce un raffinato snobismo (Costa, 2009) -, ma la sua ammirazione non 
comporta l'identificazione con le sue posizioni anche concettuali; né tantomeno c’è da appiattire la poetica di Lella Costa a quella di Franca Valeri, per il fatto che quest'ultima ne abbia curato la prefazione al suo primo libro La Daga nel Loden: nelle prime pagine della prima raccolta di monologhi di Lella Costa, la Valeri, infatti, dichiara la propria avversione per il concetto e per l'abitudine consolidata di distinguere tra maschile e femminile quando ci si riferisce al talento comico.

Eppure ritengo che Lella Costa espliciti nei suoi monologhi comici alcuni procedimenti retoric tipicamente femminili, che non tolgono nulla al suo talento e alla sua forza distintiva di comico, sia uomo o donna. Anzi si potrebbe perfino azzardare a dire che il comico, per sua natura, deve tesorizzare certe trovate e procedimenti retorici per riuscire ad accattivare il pubblico, procedimenti che richiamano uno stile che può essere tipico del genere maschile o femminile prevalentemente e che, nella loro combinazione e dosaggio, possono costruire la cifra particolare di un autore o attore comico sia uomo, sia donna. E nonostante ciò il genere dell'autore o dell'attore non sarà determinante per la propria vis comica. Credo infatti che esista un modo comico tipicamente maschile di attrarre l'attenzione del pubblico, aggressivo, invadente e scioccante: nell'ambito italiano penso a figure come Vittorio Gassman, Paolo Villaggio nei suoi sketches televisivi (quando non interpretava il suo più famoso Fantozzi) e soprattutto a Carmelo Bene in tempi più attuali e magari Ascanio Celestini. E invece, quando ricordo le battute e gli sketches di Massimo Troisi, per esempio, penso che esista uno stile di comicità femminile esercitata da uomini, e che apporta un particolare lirismo alla comicità. Nel caso della Costa, infatti, mi sembra appropriato proprio questo paragone, per la caratteristica mistura di humour, poesia e grazia che la contraddistingue. In altre parole, non distinguerei tra il sesso di persone che esercitano la comicità, quanto piuttosto ad uno stile maschile e femminile del genere "comico". Il caso della comicità Lella Costa si iscrive in questo "dosaggio" ben misurato di elementi femminili e maschili e che si manifesta nei suoi monologhi.

La prima caratteristica che colpisce nella lettura dei suoi primi monologhi è la quasi totale assenza di pause, l'asindeticità, il procedere paratattico, espediente retorico già ampiamente utilizzato nella corrente letteraria del primo Novecento, noto come il flusso di coscienza e identificato come tratto distintivo delle letterate donne. La sospensionedei periodi e della punteggiatura in un discorso, infatti, serve ad indicare una seriedi pensieri non formalizzati perché scaturiti da processi emotivi più che cerebrali, e per questo poco organizzati. E l'emotività o l'emozionalità viene ancora trattata dalla Costa come una risorsa tipicamente femminile.

La Costa d'altrondenon nega mai un chiaro orientamento di genere: c'è una dichiarata identificazione con il femminile, visto che nei suoi spettacoli descrive i tormenti e le contraddizioni dell' anima femminile che conosce bene e ci ironizza intorno. Già questa sarebbe una buona ragione di adesione e solidarietà che giustificherebbe la sua attività e scelta artistica. Ma soprattutto, la Costa, esprime una chiara scelta anche di tipo pragmatico commerciale per il suo orientamento tematico quando afferma nelle varie interviste, come in quella apparsa il 10 marzo 2010 sul sito internet http://libriblog.com/video/intervista-con-lella-costa/:

I lettori in generale sono soprattutto femminili, esattamente come il pubblico che va a teatro, e non soltanto a vedere me, è una questione di composizione maggioritaria del pubblico femminile in tutto quello che riguarda le scelte d'arte, di cultura, di letteratura, di cinema e di teatro. Penso che forse ci sia una superiore componente femminile perché la mia identità, oltretutto di monologante, è forse quella più riconoscibile.

La cifra della femminilità nei monologhi comici non si rileva solo dalla scelta delle tematiche utilizzate, che concernono l'universo femminile e i suoi problemi (vedi il riferimento ai centri di assistenza ginecologica e prematrimoniale degli anni '70 in Adlib), alla sua presa di posizione su questioni ancora vitali come ilfemminismo e l'aborto, per esempio; ma soprattutto per la forma del suo discorso.Già solo prendendo in analisi il suo libro La daga nel Loden, che si inaugura con il monologo Adlib, è evidente il ricorso alla captatio benevolentiae, tipica della retorica femminile per accattivare l'attenzione del pubblico, rivolgendosi in modo delicato, anche fisicamente, e non solo figurativamente, al pubblico, e lo fa in chiave comica paradossale, ribaltando le aspettative del pubblico e dichiarando fin dall'inizio isuoi intenti tra rivelazione di segreti apparenti e verità nascoste: 1 'attrice si presenta vestita da ballerina, in tutù, per entrare prima visivamente in "punta di piedi", con l'abbigliamento pertinente al caso, fingendo di trovarsi in un equivoco preparato ad arte:

Vi hanno avvertito che questo è un balletto classico?

Nooo! Non ve l'hanno detto.....Accipicchia!

Fan sempre così...han paura che poi la gente vada via...

Ma proprio un balletto classico di quelli noiosi, lungo, estenuante...

Che poi io non sono neanche una ballerina classica, insomma... piuttosto negata direi, sì... Vi ricordate gli elefanti, gli ippopotami della "Danza delle ore" in Fantasia di Walt Disney? Ecco, io sono uguale..

È brutto, eh?

Ma no, non è vero, va...non è vero, questo non è esattamente un balletto classico. Il fatto è che fare la ballerina classica sarebbe stato il sogno della mia vita... non l'ho mai neanche detto a nessuno quindi era difficile che ci riuscissi, però ho pensato "già che ci son lì, io mi metto il tutù, che è così femminile"

E son qua ... con quello che in realtà è uno spettacolo, uno spettacolino che ha una sua storia ormai... per esempio è nato in marzo... so, e non è un'indicazione da poco.

Perché è nato in marzo? Perché in marzo c'è il giorno 8, che in realtà c'è anche in tutti gli altri mesi, ma il giorno 8 marzo è particolarmente significativo, perché come sicuramente ricorderete è la giornata della donna.

Lo sanno tutti, credo ci sia anche la giornata internazionale del panda e in genere tutte le specie in via di estinzione, quindi noi abbiamo questa giornata ma veramente tutta per noi possiamo fare un sacco di cose, ci danno i fiori, ci portano a ruzzare nei prati... bellissimo (Costa, 1993:15-16)

In seguito, l'attrice si toglie gli abiti della ballerina classica -che nell'immaginario collettivo connotano delicatezza e poesia-, e cioè la fascia di tulle rosa dalla testa, le 
scarpine da mezzapunta rosa, la fascia di tulle rosa dalla vita, si toglie il corpetto nero con uno strappo, infila un paio di scarpe d'argento con tacco a spillo, e si ritrova addosso un abitino attillato a disegni leopardati -abbigliamento più aggressivo e provocante, consono per il tema da affrontare con spirito "graffiante" - per meglio affrontare temi e riferimenti più aggressivi nel suo monologo. $\mathrm{E}$ lo fa intonando una canzone sensuale e accattivante, This guy's in love with you di Burt Bacharach, adattandola nella versione personale This girl's in love with you con voce altrettanto graffiante, per introdurre la questione scottante dell'Aids in Italia e dell'approccio mistificato al tema ancora alla fine degli anni '80. La pertinenza della maschera (gli abiti) e della retorica (i discorsi e la loro struttura) nella nostra autrice, dunque, è ben esplicitata figurativamente nei suoi spettacoli a partire dai monologhi pubblicati in Daga nel Loden. Questa "maschera" di femminilità, di passione dichiarata per le frivolezze -come la sua collezione di scarpe con il tacco a cui dedica una vetrina particolare nel salone della sua casa-, non esclude il forte impegno politico che anima la scelta di questa carriera artistica come dichiara nell'intervista rilasciata a Roberto Carnero per L'Unità, dell' 8 dicembre 2009:

\begin{abstract}
Sono arrivata a fare l'attrice, il mio grande sogno - ci racconta - piuttosto tardi, dopo una stagione di impegno politico a sinistra. Erano anni, il decennio dei '70 i primi ' 80 , in cui si credeva alla politica come assunzione di responsabilitì verso la collettività. Questa militanza politica e culturale è stata la mia formazione. i co colne. utilizzare questo mio lavoro anche per fare qualcosa di buono per gli altri.
\end{abstract}

La retorica della Costa è femminile quando fa digressioni, divagazioni o addirittura salti apparentemente poco coerenti rispetto al tema del discorso principale, trasgredendo a linearità logica, spesso considerata sinonimo di affidabilità e per convenzione attribuibile alla mente maschile. Questo si deve alla tendenza del pensiero femminile che si elabora sul modello del "link" multimediale, sempre a cavallo "tra" temi diversi che hanno tra loro delle associazioni o comunanze labili, legati ad un significato particolare, un ricordo (Vedi a questo proposito il monologo Malsottile dedicatoa questo tema) o talvolta solo ad un suono. A ciò si deve, infatti, il ricorso comico derivante dalle assonanze e sulle paronomasie, parole associate per meri spostamenti vocalici, seguendo un filone di comicità postmoderna quasi demenziale come esprime nel monologo Coincidenze (p. 76), dove adatta la famosa canzone di Sergio Endrigo, Per fare un tavolo, intonandone la melodia con un testo giocato sul suono e la parodia dei nomi commerciali dei sedativi più conosciuti -e, per associazione, dei loro consumatori abituali-:

Per fare un Tavor

Mi dai due Valium

Se non c'è il Valium

Va bene il Noziman

Se non c'è il Noziman puoi darmi un Serpax
Se non c'è il Serpax

Va bene il Mandrax

Se non c'è il Mandrax posso farmi un Halcion

Mi faccio un Halcion

Mi faccio un Halcion

Mi faccio un Halcion ma vorrei un TA-A-Vor..

Dal punto di vista linguistico la sua caratteristica comica si effonde attraverso i motti di spirito e i giochi di parole: ad esempio, nel corso del suo monologo Coincidenze, ad ogni menzione dell'espressione "Weltanshauung", si sovrappone, come una colonna sonora, la canzone dei Beatles, Twist and Shout (Lepri, 1996: 38-44). Oppure per citare esempi dal suo monologo Adlib, quando l'autrice ironizza sull'uso di un linguaggio collettivo poco familiare tipico degli anni 70-80:

$<\ldots>$ Il capitolo più irresistibile era quello che noi chiamavamo degli strafalcionari: una specie di fior da fiore di tutte le gaffes, gli strafalcioni appunto, che i nostri amatissimi compagni si lasciavano sfuggire soprattutto in quegli anni...

Era una roba scientifica, divisa per paragrafi, a seconda degli argomenti.

C'era il genere "vacanze intelligenti" supplemento dell'“Espresso": " Si perché noi si è stati ora a Firenze per vedere questa mostra degli affreschi del Caporetto..."

Quelli che di lì a pochi anni sarebbero andati a vedere i bronzi di Versace,

suppongo...

Poi quelli della proprietà di linguaggio a tutti i costi "la parola giusta al momento giusto": "No, perché, Carlo è simpatico, no, simpatico è simpatico, però quello parla, parla, parla troppo, secondo me è un vero gonorroico..."

E gli alternativi? "Guarda io ne ho piene le palle del turismo di massa, cioè ti rend conto, noi siamo stati ora in Africa no, non con un viaggio organizzato, ma veramente liberi, cioè abbiamo veramente vissuto a contatto con la gente nel cuore del continente nero, in questi villaggi poverissimi, cioè ma ti rendi conto, là la vita è tremenda... là la gente vive ancora allo stato ebraico...

$<\ldots>D^{\prime}$ altra parte invece abbiamo i compagni sindacalizzati sino alla morte... quelli che ti dicono:

"No, perché qualunque cosa succeda io non sarò mai convivente con il padrone". (Costa, 1993: 46-47)

La sua attività di attrice brillante è quasi oscurata dalle mancate presenze negli ultimi anni sullo schermo televisivo che sembra essere l'unica vetrina di diffusione $d$ notorietà degli autori, attori e personaggi teatrali. Il mondo del teatro e i suoi circuiti tendono ad allontanarsi dalla popolazione di massa che accede solo alla televisione e per lo più, in misura minore, al cinema. In questo modo, il teatro attuale, in qualche modo si seleziona un proprio pubblico a cui si rivolge preferibilmente, come dei "target" di utenti: c'è il genere classico, il genere popolare vernacolo, il genere cabarettistico, il genere leggero, il genere musical, ecc. Anche Lella Costa, escludendosi dalle ospitate televisive, pretende, "inventa" e si "costruisce" un proprio pubblico intelligente,colto e ironico, un pubblico che sa riconoscere la propria identità incerta, le manie,le contraddizioni e le occasioni perdute e cerca stimoli e idee per trovare soluzioni. Allo stesso tempo, questo stesso pubblico, seguendo e frequentando il "personaggio" 
Lella Costa, si identifica con lei ideologicamente e si schiera, insieme alla sua paladina, contro i "dictat" televisivi e della sua cultura omologante e riduttiva.

Nell'intervista pubblicata su L'Unità del 8 dicembre 2009, la Costa, alla domanda su un capitolo del suo ultimo libro La sindrome di Gertrude, Rizzoli, 2009 dedicato alla "Televisione" -ambito in cui ha anche coltivato esperienze e che negli ultimi tempi giudica condizionata e condizionante - chiarisce la sua posizione:

Nella mia carriera di attrice, ho fatto poca tv e molto teatro. E oggi sono felice di Never placet, condizionat dal potere politico oltre che dalle loro faide interne. Che non mi chiamino a fare tv non è grave, perché in fondo questo non è la mia vocazione. Trovo invece grave che professionisti del piccolo schermo come Serena Dandini, Fabio Fazio o Milena Gabanelli non sappiano da una stagione all'altra se il loro contratto verrà rinnovato. La tv pubblica non può sprecare così le risorse che ha al suo interno.

\section{Una giullare "attivista"}

Del giullare Lella Costa ha la forma, la maschera, ed anche la retorica. Tuttavia non si può dire che -come vuole la tradizione dei giullari e dei commedianti dell'artel'attrice improvvisi nei suoi spettacoli. I suoi monologhi e i relativi spettacoli sono molto misurati, molto studiati, non si lascia quasi mai nulla all'improvvisazione. E nonostante ciò, l'effetto è di una grande spontaneità. Come dichiara nell'intervista rilasciata a La Stampa il 31 ottobre 2009:

Non salgo in palcoscenico se non ho scritto tutto. Poi, certo, le parole scritte devono diventare teatrali e viventi. L'improvvisazione per me è quasi inesistente. Nel caso, so precisamente dove può accadere, senza togliere ritmo e disegno emotivo al testo. Poi ci sono gli imprevisti e gli incidenti dal vivo, certo. In quei casi, anche se la reazione improvvisata funziona, è importante non provare mai a replicarli. Non funzionerebbero più.

Lella Costa è colta, apparentemente "snob", la sua letteratura è decisamente intrisa di cultura alta, ma non ne presume, anche se la diffonde. È stata per lungo tempo doppiatrice della soap operastatunitense Sentieri e nei suoi spettacoli, come un vero giullare, opera contaminazioni continue tra cultura alta e bassa. I suoi monologhi sono farciti di riferimenti che provengono dalle tradizioni più eterogenee: da Walt Disney a Wilhelm Reich, da Tom Waits a Louise Mary Alcott, da Christa Wolf a Saint Exupery, da Paolo Conte ai Pooh a Gabriel García Marquez, ai nomi delle serie televisive più popolari e delle soap opera, che, come sostiene l'autrice, è capace di produrre anche piccole mitologie generazionali come Rin Tin Tin, o Giovanna, la nonna del corsaro nero. (Lepri, 1996: 38-44). Da questa contaminazione tra letteratura e cultura "alta" e "bassa", di tradizione antica nel genere "umile", dalla convivenza di stimoli, codici letterari e non, e conoscenze così eterogenee, nasce la sua comicità grottesca e pertanto fortemente parodica e ironica, un po' trasognata e surreale e molto poetica, di una poesia che si fonda su elementi fortemente reali e concreti e sulle situazioni contrastanti e paradossali.

\section{RIFERIMENTI BIBLIOGRAFICI}

Alessi, Ch., Tra scrittura e scritture. La drammaturgia italiana al passaggio del terzo millennio, 2005, tesi di laurea pubblicata da www.dramma.it

Bene, C., "Il monologo", Opere, Milano, Bompiani, Milano, 2002.

Carnero, R., L'Unità, 8 dicembre 2009.

Costa, L., La daga nel Loden, Milano, Feltrinelli, Milano, 1993.

----, La sindrome di Gertrude, Milano, Rizzoli, 2009.

Geis, D. R., Postmodern Theatricks: Monologue in the Contemporary American Drama Michigan, The University of Michigan Press, 1993.

Lepri L. "Donne che si prendono in giro", Tirature '96: comicità, umorismo, satira, parodia, a cura di Vittorio Spinazzola, Milano, Baldini \& Castoldi, 1996.

L'Unità, 8 dicembre 2009: Collegamenti internet: http://www.irmaspettacoli.it/lella_ autrice.html

http://libriblog.com/video/intervista-con-lella-costa/

Martín Clavijo, M., El monologo en el teatro de Stefano Benni, Sevilla, Arcibel, 2009.

Portone, F., "Il corto teatrale: la drammaturgia e il teatro di oggi" in Dramma, http:www. dramma.it; http://www.dramma.it/articoli/articolo120.htm; ultimo aggiornamento: 14 maggio 2008

Puppa, P., "Il teatro del racconto: il caso Paolini”, Granteatro: Omaggio a Franca Angelini, a c. di Beatrice Alfonzetti, Daniela Quarta e Mirella Sandini, Roma, Bulzoni, 2002.

----, Il teatro dei testi, Torino, UTET, 2003. 